\title{
The Curse of Lymphatic Filariasis: Would the Continual Use of Diethylcarbamazine Eliminate this Scourge in Papua New Guinea?
}

\author{
Anne Lanham, Lillian Mwanri* \\ Discipline of Public Health, School of Medicine, Faculty of Health Sciences, Flinders University, Adelaide, Australia \\ *Corresponding author: lillian.mwanri@ flinders.edu.au.
}

Received December 17, 2012; Revised January 19, 2013; Accepted February 28, 2013

\begin{abstract}
Lymphatic filariasis (LF) is a significant public health problem across the developing world and is highly prevalent in Papua New Guinea (PNG) with 16 out of 20 provinces recorded as endemic areas. This paper provides a comprehensive overview and discussion of various strategies to eliminate LF, explores the use of appropriate antifilarial drugs and examines the ways they can be taken to develop the most effective method for PNG. A systematic search of English literature was conducted using the National Library of Medicine (PubMed) and Science Direct databases between 1980 and 2012. Due to lack of high level research evidence, narrative as opposed to systematic review was conducted to identify major emerging themes. More than 1.3 billion people in 72 countries including PNG are threatened by LF. Over 120 million people worldwide are currently infected, with about 40 million disfigured and incapacitated. In 2000, the PNG National Department of Health (NDOH) began preparatory studies for a national program for LF elimination, but lack of commitment and funding has curtailed any significant progress and the disease remains highly prevalent in this country. Challenges and opportunities for elimination of LF in PNG exist and the authors predict that a combination of methods specifically tailored for PNG will have the greatest chance of success. There is an emphasis on calling for more research in a range of areas but this should not delay or compromise the introduction of a nation-wide LF elimination program or deny access to treatment for those afflicted with LF.
\end{abstract}

Keywords: Papua New Guinea, lymphatic filariasis, Wuchereria bancrofti, DEC salt, vector control, elimination

\section{Introduction}

Lymphatic filariasis (LF) is a major infectious disease and one of the neglected public health problems of the 21 st Century. It is caused by three species of nematodes: Wuchereria bancrofti, Brugia malayi and Brugia timori [1]. All infections in Papua New Guinea are caused by the parasite Wuchereria bancrofti which accounts for $90 \%$ of the world's cases [2], andshares the same vectors that transmit malaria, mainly Anopheles punctulatus, and less frequently Anopheles farauti and Anopheles kolinensis [3]. The adult Wuchereria bancrofti worms live in groups (called "nests") within the human lymphatic system with a long life span of 4-6 years. After mating, they release thousands of microfilaria $(\mathrm{mF})$ into the blood stream of the host and these are picked up by the vector mosquitoes [4,5]. Microfilariae do not reproduce in the vector but rather each worm completes two intermediate larval stages (L1 and L2) andmolts to become a third stage (L3) infective parasite [4,5]. It is transferred to the human host when the mosquito takes a blood feed where it migrates to the lymphatics and becomes an adult worm. The diagrammatic representation of LF life cycle is presented in Figure 1 below [6].

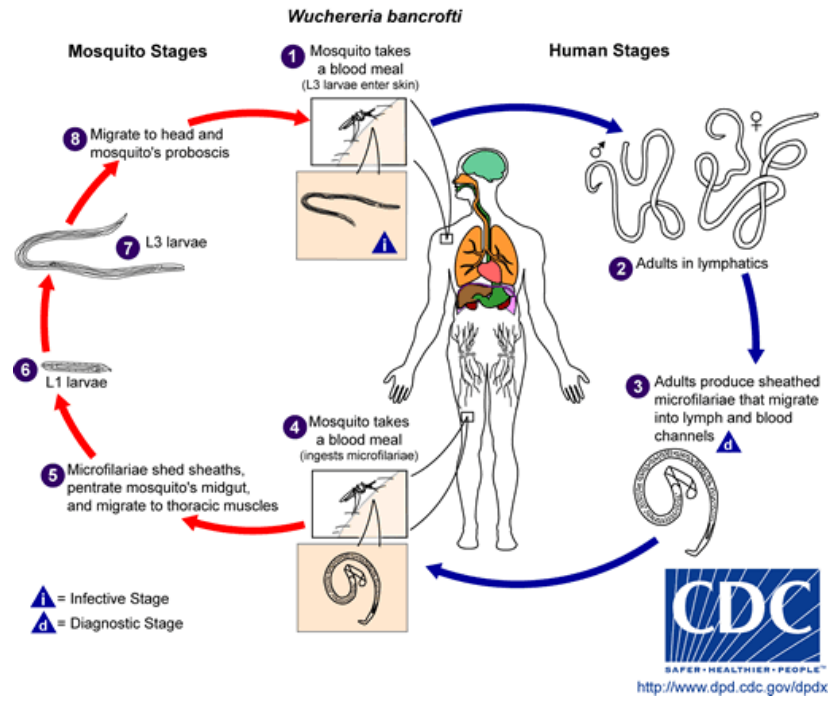

Figure 1. Life Cycle of Wuchereria bancrofti

(Source: Centers for Disease Control and Prevention).

The parasite causes immunosuppression in the victim which is especially significant in the case of children as the parasite constantly attacks the respiratory and renal systems eventually causing significant damage [5,7]. 
It has been reported that more than 1.3 billion people in 72 countries including Papua New Guinea (PNG) are at risk [7]. Evidence demonstrates that this is an endemic public health problem in PNG [5,11]. The Sero-prevalence surveys conducted by the PNG Department of Health in 5 years from 2000-2004 showed that over 4.4 million people in 71 out of 85 districts in 16 out of 20 provinces of the country, including the National Capital District, are at risk $[3,7]$.

Lymphatic filariasis causes a wide spectrum of clinical and sub-clinical disease with a range of symptoms and adverse effects. The most common and obvious symptom of lymphatic filariasis is elephantiasis (an oedema with thickened skin and underlying tissues) [2]. Approximately two-thirds of infected individuals show no overt evidence of disease, but when tested, demonstrate some degree of parasite-associated immunosuppression and many show evidence of renal dysfunction [11,12]. Research shows that the damage done by this parasite has been understated as people with visible signs of the disease make up only a small percentage of those affected in endemic areas $[5,13]$. In PNG, many people, including government officials, believe that it is not a significant disease as only a third of those affected have the external signs of disability and disfigurement including swelling of breasts, sexual organs and limbs. The immunochromatographic rapid card test (ICT AMRAD, Sydney, Australia) used in East Sepik Province reported a prevalence rate of infection up to $98.2 \%$ the highest in the world and there is evidence that the global burden of filariasis is increasing $[5,12,14]$.

Haddix \& Kestler (2000) acknowledge that LF is the disease caused by poverty and a disease that perpetuates the poverty cycle [15]. This disease causes enormous physical, social, psychological and economic suffering, currently infecting more than 120 million people with about 40 million disfigured and incapacitated [2,7].

The International Task Force for Disease Eradication in 1993 classified LF, along with five other infectious diseases, as potentially eradicable. In 1997, a resolution adopted by the World Health Assembly called on Member States to initiate steps to eliminate LF as a public health problem. The first regional filariasis elimination program was formed in 1999 and was an alliance of 22 Pacific Island nations and territories called PacELF (the Pacific Programme to Eliminate Lymphatic filariasis). The twin goals of PacELF are to stop transmission of filariasis and to alleviate the suffering caused by the disease. WHO launched the Global Programme to Eliminate Lymphatic Filariasis (GPELF) in 2000 which has joined with other international bodies to become the Global Alliance to Eliminate Lymphatic filariasis (GAELF) [5,15].PNG faces significant challenges to meet PacELF goals. A high proportion $(87 \%)$ of PNG's people live in rural areas in widely scattered communities that are often not accessible by road $[3,7,16,17]$. The prevalence of lymphatic filariasis in PNG is amongst the highest in the world reaching $100 \%$ in some locations [17]. Projects including annual Mass Drug Administration(MDA) have been undertaken over many years with little long term success. PNG is the only endemic country in the Pacific region that has not yet introduced a countrywide program to eliminate LF [18]. PNG is on the PacELF schedule and plans have been developed but no implementation at a national level has taken place.

\section{Methods}

A systematic search of English literature was conducted in March 2012 using the National Library of Medicine (PubMed) and Science Direct databases between 1980 and 2012. Keywords used included: DEC salt and lymphatic filariasis Diethylcarbamazine and PNG, Diethylcarbamazine and $\mathrm{LF}$ and PNG, MDA and filariasis and PNG and Lymphatic filariasis and PNG. Google search was also conducted to identify other work done on LG in PNG. All titles and/or abstracts were screened to identify original publications. Hand search to include PNG Government reports and other relevant documents were also conducted. A total of 65 articles were identified as summarised in Table 1 below.

Table 1. Literature Search strategy and outcomes

\begin{tabular}{|c|c|c|c|c|c|}
\hline Date & Databases & keywords & \multicolumn{2}{c|}{ Wave 1} & \multicolumn{2}{c|}{$\begin{array}{c}\text { No. Selected } \\
\text { Full }\end{array}$} \\
\hline $18 / 3 / 12$ & Pub Med (Medline) & DEC Salt or diethylcarbamazine and LF and PNG & 88 & Reviewed all & 5 \\
\hline $22 / 3 / 12$ & Science Direct & DEC salt or diethylcarbamazine and LF and PNG & 60 & Reviewed all & 5 \\
\hline $24 / 3 / 12$ & Google Scholar & LF, PNG, Diethylcarbamazine and PNG & 19 & & \\
\hline $1-24 / 3 / 12$ & Previously extracted & Diethylcarbamazine and PNG & & \\
\hline $1-24 / 3 / 12$ & Grey literature & PNG Government plans and reports, other research & & \\
\hline & TOTAL & 6 & & \\
\hline
\end{tabular}

The search was extended by scrutinizing the references of selected articles and only articles for which the full text and abstract was available were considered. Due to the paucity of high quality information the search was extended by scrutinizing the references of selected articles to identify additional studies that may have been older than the timeframe stipulated. Papua New Guinean Government plans and reports and other relevant documents were also reviewed. Because of the methodological complexity and limited number of articles providing high level of evidence, narrative including content analysis of articles as opposed to systematic review has been conducted [19]. Major emerging themes were identified through recurrence in literature as well as using our experiences and knowledge of public health related to this subject. Details of these themes are described below:

\section{Results}

\subsection{Strategies Available to Eliminate Lymphatic Filariasis} 3.1.1. Community Awareness, Education, Testing and
Surveillance 
Any program to eliminate LF needs the participation and cooperation of the wider community. There needs to be community awareness and education programs throughout the country informing people about the extent of the burden of disease in physical, psychological, social, economic aspects and an awareness of the program itself to secure maximum compliance and support from communities. Studies in PNG have shown that there is a very low level of knowledge of the disease or its cause outside the people who are directly affected. Information should include the testing regime, the MDA, the possible adverse effects and the follow up involved in this program. Community leaders will need to be identified to provide support to the official personnel who will be involved in the project. Research has found that community directed MDA programs achieved much higher levels of coverage than those delivered exclusively through the formal health sector and were especially effective where health facilities were limited $[3,20]$.

People in PNG are very accepting of the National Immunisation Program and take great care of each family member's medical record book. PNG NDOH undertakes annual SIA (Supplementary Immunisation Activity) throughout all provinces to ensure full immunisation coverage and where attendance is compulsory. This would provide the perfect vehicle for the one round of MDA with DEC and Albendazole (tablets provided free of charge by the manufacturer) and DEC salt, sufficient for 6 months, could be distributed as an additional incentive.

In the evaluation of the initially successful five year MDALF control program in Samarai Murua District, PNG, using DEC, it was noted that "the importance of effective community consultation and education programs cannot be overemphasised" [17]. Costs for this five year LF program were minimal because of involvement by local people in the distribution of DEC.

\subsubsection{Mass Drug Administration using DEC and Albendazole or DEC and/or Ivermectin}

Diethylcarbamazine (DEC) has been used as an antifilarial drug since 1947 [13]. The current dose of DEC for treatment is $6 \mathrm{mg} / \mathrm{kg} / \mathrm{day}$ for 12 days up to a total dose of $36-72 \mathrm{mg} / \mathrm{kg}$ of body weight $[21,23]$. The World Health Organisation calls for the distribution of community wide annual Mass Drug Administration (MDA) of single doses of albendazole plus either DEC or ivermectin to interrupt transmission of the parasite for 4-6 years [2,7]. The second goal of the WHO program is to alleviate the suffering caused by lymphatic filariasis through morbidity management and disability prevention.

The strategy recommended by the WHO of annual treatment with two-drug combination has proved to be safe. The LF program is arguably the most effective "propoor" public health program currently operating which is based on country commitment and partnerships supported by a global program and alliance [24], Premise of the Global program is that once the antigenaemia prevalence in the 6-10 age group throughout the country falls below $0.1 \%$, transmission will be interrupted permanently [25].

A three year MDA program was undertaken recently in a number of rural villages near Madang, PNG, with some promising results [26,28]. There was a significant reduction in all filariasis infection parameters in the study village with good compliance but researchers claim that prospects would be brighter if the MDA included a distribution of insecticide treated bednets [25].

In an earlier trial in 1994 in Madang, reported in 2002 with 2500 residents [9], the effects of four annual treatments with single dose of diethylcarbamazime plus invermectin or diethylcarbamazime alone were assessed. The findings suggested that eradication may be possible in areas with moderate rates of transmission but longer period of treatment may be necessary where higher rates prevailed. In a similar study in an area in India with high infection levels, 10 years of MDA using either diethylcarbamazime or invermectin showed that the DEC alone was more effective than the ivermectin alone with the $\mathrm{mf}$ rate declining to $<1 \%$ in some villages [41].

Follow up studies of MDA are yet to show a complete interruption of transmission of microfilaria, though the infection rates are reduced to a very low level [21]. All the studies seem to indicate significant reduction using one drug and a slight increased benefit in using two different drugs, but unless the final rate is $<1 \%$, the risk of returning infection seems likely.

\subsubsection{DEC Salt}

Salt is the ideal vehicle for introducing a regulated amount of a substance into a widely used food. It is universally consumed in fairly consistent amounts, is stable, inert and travels well.

DEC was introduced into salt as filariasis control in the 1960 s by a group of researchers in Brazil who undertook a number of pilot studies in humans using a male prison population $[27,28]$. The optimum concentration of DEC in salt was established at $0.4 \%$.

China began a national control program for LF in 1956 using DEC but didn't commence DEC salt until 1970s $[27,29]$. By then, the prevalence rates were low $(9-13.3 \%)$ and salt at concentrations of $0.24-0.3$ were introduced for 6 months. Through to 1988 when China claimed transmission of LF was eliminated, about 190 million were treated. No adverse effects were mentioned in reports from China and details of the organisation of programs are not available. Availability of the salt was controlled by the government ensuring very high coverage of the population.

A number of studies on the proposed use of DEC-salt have been produced including the proposal to the PNG government by Trevor Milner. His report details all the necessary steps needed to implement a DEC-fortified salt program in PNG, including costing [3].

In a detailed review of 21 studies of DEC medicated salt programs, it was shown that DEC kills microfilaria but repeated doses are needed before adult worms are killed or sterilised. The review also found that DEC may be more effective when given in a very low dose continuously over a long duration (such as six months) than in higher doses at one point in time such as MDA [31].

A school-based health education campaign is suggested as a potential tool for social mobilisation to promote DEC salt [21]. Through surveys and questionnaires, researchers established that information received through school students and teachers conferred credibility and was a strong motivating factor for members of the community to use DEC salt. 


\subsubsection{DEC Treatment of Positive Cases}

With some successful programs, extensive government funded education and awareness programs have been in place for many years. After the discovery of DEC in 1947, Japanese scientists began studies to look at its use in disease elimination and in the following years undertook a range of surveys and mass testing for filariasis. It wasn't until 1978 that elimination of LF was achieved [32]. The Japanese strategy was mass screening of the whole population using blood smears, DEC treatment for positive cases, $6 \mathrm{mg} / \mathrm{kg}$ once a day for 12 days supplemented by spraying with DDT or malathion as vector control.

Low population density and difficult terrain make mass screening and treatment in PNG a very difficult option. While there is a sufficient DEC in Port Moresby (the capital city of PNG) to treat all diagnosed cases of LF, poor and unreliable distribution to rural and regional centres to commence and maintain treatment has resulted in excessive suffering for those affected with LF and ongoing transmission of the disease.

\subsubsection{Vector Control: Long Life Impregnated Nets, Pheromones and Indoor Residual Spraying}

More than 70 species of mosquitoes within six different genera are known vectors of $\mathrm{W}$. bancrofti including Anopheles spp., Aedes/Ochleratatus/Downsiomyia spp., Culex spp and Mansonia spp.. Among the anophelines, 36 species are capable of both malaria and LF transmission, 26 of which are regarded as major LF vector species. In PNG, there are 9 vectors of $\mathrm{W}$. bancrofti and 4 vectors cotransmitting.

The effectiveness of the prevention of infection with $\mathrm{W}$. bancrofti in endemic areas by vector control programs has been noted to differ greatly according to the species of vectors involved in the transmission [9]. Wayne Melrose in his article of 1999 warned about possible increase in LF vectors due to recent increased deforestation [33]. Vector control was once advocated as the primary tool to control filariasis because effective antifilarial drugs were unknown. W. bancrofti was eliminated from Australia primarily by sanitation campaigns against Culex quinquefaciatus [34].

Although the majority of programs aimed at interrupting transmission of $\mathrm{W}$. bancrofti by vector control alone have been ineffective, a notable exception was the Solomon Islands where anopheline-transmitted W. bancrofti was unintentionally eradicated as part of the residual insecticide campaign of the malaria eradication program [35].

Pheromones have long been known to be important to the lives of insects in mating and scientists used pheromones to disrupt the mating procedures of the Beet army-worms, a serious pest in the cotton producing areas of the USA in 1997, and saved the crop [36]. The producation of synthetic sex pheromones has led to more research on using this technique for vector control in developing countries targetting sandflies in South and Central America. Results of the first study to show attraction of a human disease transmitting insect to a synthetic pheromone in the field may lead to developing new tools in vector control which would be invaluable particularly if applied to Anopheles spp. Mosquitoes [37].
Unfortunately, vector control has been relegated to a secondary role [38] although Long Life Impregnated Nets (LLIN) and indoor residual insecticide (IRS) campaigns have proven effective in reducing filarial transmissions in the past [39].

Spraying with DDT was once widely used for vector control in malarial areas. Use of DDT is now strictly controlled under the Stockholm Convention (2004) and can only be used for disease vector control according to the recommendations and guidelines of the WHO [39]. The WHO recommends only indoor residual spraying (IRS) (spraying only on the inside walls of buildings) of DDT for disease vector control.

There has been a massive scaling up of the free distribution of long-lasting insecticide treated nets in many countries including PNG where there has been significant coverage in many provinces. This is a welcome public health initiative protecting the community against both LF and malaria.

Testing for LF antigenaemia using ICT is now widely used and is "an ideal tool for surveys" [5]. Whichever strategy to eliminate LF is chosen, pre and post testing to establish $\mathrm{mF}$ levels is necessary as well as long term surveillance to ensure elimination of LF transmission.

\subsection{Papua New Guinea National Department of Health Plans and Implementation}

Since 2000, the PNG Department of Health (NDOH) began preparatory studies for a national program for LF elimination and in 2005 began implementing a 15year National Strategic Plan to eliminate Lymphatic filariasis 2004-2020 (PNGELF). Although the plan is comprehensive and the budget of US\$79million realistic, implementation is lagging. Some endemic provinces have not received one round of MDA. Any community with a single case of lymphoedema or hydrocele meets the NDOH criteria for inclusion in an MDA program [41].

In 2007, the plan was revised because of slow implementation and inadequate coverage of MDA. People are not returning for second and third year dosage because people with $\mathrm{mf}$ suffer adverse reactions to the drugs. PNG authorities are looking for alternate strategies that may better suit the PNG environment $[2,14]$.

\section{Discussion}

Lymphatic filariasis is a severe public health problem affecting a significant proportion of population in PNG. The selection of the most appropriate strategy to eliminate the transmission of lymphatic filariasis, whether it is mass drug administration, DEC-fortified salt, annual single dose of albendazole and DEC or a combination of strategies depends on a number of factors. Past experiences with similar public health programs such as salt iodization, national vaccination programs can help to predict the success of a LF campaign.

A number of studies have shown that DEC alone does not kill or sterilise all W. bancrofti adults and some have acknowledged that MDA campaigns may fail to maintain adequate treatment coverage to achieve lymphatic filariasis elimination [35,42], Many have advocated for the inclusion of vector control as part of the campaign as 
there is evidence that vector control successfully eliminated lymphatic filariasis when implemented alone or with MDA [40]. It is suggested that integration of mosquito control as part of the PacELF campaign would require additional basic ecology research on the important vectors [42]. For example, in South India, ten rounds of DEC alone was administered over a nine year period, bringing about significant falls in the microfilaria prevalence and the vector infection rates. Treatment coverage ranged from $49 \%$ to $84 \%$ reducing the prevalence from $14.5 \%$ to $2.44 \%$ [43].

The Pacific region has several reasons to feel vulnerable about re-emergence of the disease. The persistence of LF in the Western Pacific, despite several MDA campaigns, urges some caution to the hope that an MDA campaign alone will eliminate filariasis and there are calls for consideration of DEC-fortified salt $[3,35,44]$. There are a number of historical reports including one on Maupiti, an isolated island community in French Polynesia, which participated in mass treatment campaigns and prevalence surveys for more than 34 years because there are often difficulties with non-compliance [45].

Evidence exists that shows the difficulty of eliminating macrofilaria (adult worms in situ). For example, in a random trial to assess directly the effect of various doses of DEC on adult W. bancrofti, 31 infected men received doses from $1 \mathrm{mg} / \mathrm{kg}, 6 \mathrm{mg} / \mathrm{kg}$, or $12 \mathrm{mg} / \mathrm{kg}$. Beginning 7 days later, the dosage and duration were progressively increased. Findings were that above $6 \mathrm{mg} / \mathrm{kg}$, the macrofilarcidal effect of DEC did not increase with dose and a significant portion of adult $\mathrm{W}$. bancrofti were not susceptible to DEC during the study period [46].This is supported by Burkot and Ichimori who claim that the primary obstacle for an MDA based strategy to eliminate filariasis is the uncertainty of the DEC plus albendazole treatment in eliminating adult worms [42].

Drawbacks of DEC MDA are that locating each and every resident in a particular area is inherently a difficult and time-consuming operation; that current recommendations for tablets exclude pregnant women and children under 2 years and when individuals experience side-effects, they are reluctant to return for the next round of drugs. There are reports from Brazil, China, Tanzania, India and Japan to suggest significant reduction in LF by way of salt fortification with DEC. These studies point out that DEC fortified salt may have both micro-filariacidal and macrofilariacidal properties with long-lasting beneficial effects. Salt fortification has shown results in 1-2 years similar to the end of 4-6 years of MDA, with minimal side effects [47].

Challenges of eliminating LF exist in PNG. For example, there are only 4 provinces out of 20 that are considered non-endemic and one of the most significant questions asked is whether every province should be included in the MDA. Additionally, PNG has a population which is highly mobile and mosquitos have no respect for borders. Much of PNG is remote and inaccessible with few roads and poor communication. Providing drugs on a regular basis is almost impossible and those who experienced unpleasant side-effects are unlike to return for follow up treatment. It is plausible to postulate that a program that includes all residents of PNG would provide a better chance of eliminating transmission of LF.

\subsection{Future Direction}

A systematic review of 57 randomised studies of drug efficacy for Lymphatic filariasis concluded that the administration of multiple drug regimens (or DECmedicated salt) is the most effective means of lowering $\mathrm{W}$. bancrofti microfilaria [48]. It was stated that there is a continuing need for large conclusive studies comparing the efficacy of drug regimens and calling for more research to fill this existing gap in knowledge at this time in the interest of affected populations and the GPELF partnership.

Every research study and review concludes with a statement that more research is needed in some particular area. We argue however that, this is not a good reason enough to preclude the planning and the commencement of the nation- wide program to eliminate LF in PNG immediately using DEC. Any gaps can be individually addressed as they appear and more DEC made available. This was the strategy adopted by the Chinese authorities who were eventually successful through continued surveillance for many years, eliminating small outbreaks as they presented.

A coordinated approach using a number of the strategies outlined is the only way that LF can be successfully eliminated in PNG. The essential elements would be one round of MDA (DEC and Albendazole) with immediate introduction of DEC-fortified salt for a period of 1-3 years combined with integrated vector management. The DEC salt would be the only salt available in the country so that importers would have to be advised and protected by legislation. It is suggested that the one round of MDA would coincide with the SIA (Supplementary Immunisation Activity) when everyone reports to the health centres with their children and the salt would be given out free to all those who took their tablet. Replacement of LLIN would be offered every two years and the supply would have to be consistent as sleeping under nets affords the children no opportunity to develop an immunity to the parasitic diseases.

The four provinces considered non-endemic would benefit from the DEC-fortified salt and the integrated vector management but would not be included in the MDA unless further testing indicated otherwise.

This program is essentially a combination of the" National Strategic Plan to Eliminate Lymphatic Filariasis 2004-2020 (PNGELF)" and "an Investigation into the feasibility of a National Program of DEC Salt for Papua New Guinea for the Elimination of the Transmission of Lymphatic Filariasis" [3]. From examination of previous LF elimination programs, the combination of one round of MDA and the introduction of DEC-fortified salt should provide best opportunity to decrease the numbers of macrofilaria and microfilaria and would eliminate intestinal parasites. The introduction of the DEC-salt program at year one of the proposed MDA would reduce the proposed costs of US\$79million considerably, would result in elimination of LF transmission earlier and would have more community compliance.

The program would start immediately with extensive community awareness and education. At the same time, legislative and regulatory changes would need to be approved by the PNG parliament to introduce DECfortified salt as the only salt available in the country. 
There would follow an extensive testing regime to establish the baseline level of LF antigenaemia and microfilaraemia before the MDA is undertaken. Some of this preliminary testing has possibly already been completed. At the time of the MDA, LLIN would be distributed if required and indoor residual spraying would be considered in some areas. Any people exhibiting symptoms of LF would be referred for treatment under the Disability Prevention and Control part of the program. DEC salt would be distributed with the MDA and would continue until surveillance testing indicated that LF transmission had been eliminated. Integrated control strategies targeting LF and malaria in areas sharing the same vector species would be a very cost-effective approach [4].

To counter the possible increase in vectors due to deforestation by timber and extractive companies, regulations must be introduced to delegate responsibility for vector management to the developer as part of the approval process for major developments.
Successful elimination of LF has only been accomplished with detailed preparation, an informed, supportive population and a determined and committed government/authority to complete the task by active surveillance over many years. Implementation of any nation-wide program in PNG would require political will and commitment from the PNG government as well as significant financial and personnel resources from international organisations.

In regards to the second part of the LF plan, alleviation of the suffering caused by the disease can begin immediately. As the drugs have been provided free by the manufacturers, distribution to endemic areas should be hastened and with proper treatment, some of the symptoms that people are suffering can be reversed. Follow up- investigations in China indicated that the acute manifestations disappear or greatly improve after a 6 month filariasis eradication program by low dose DEC medicated salt [49].

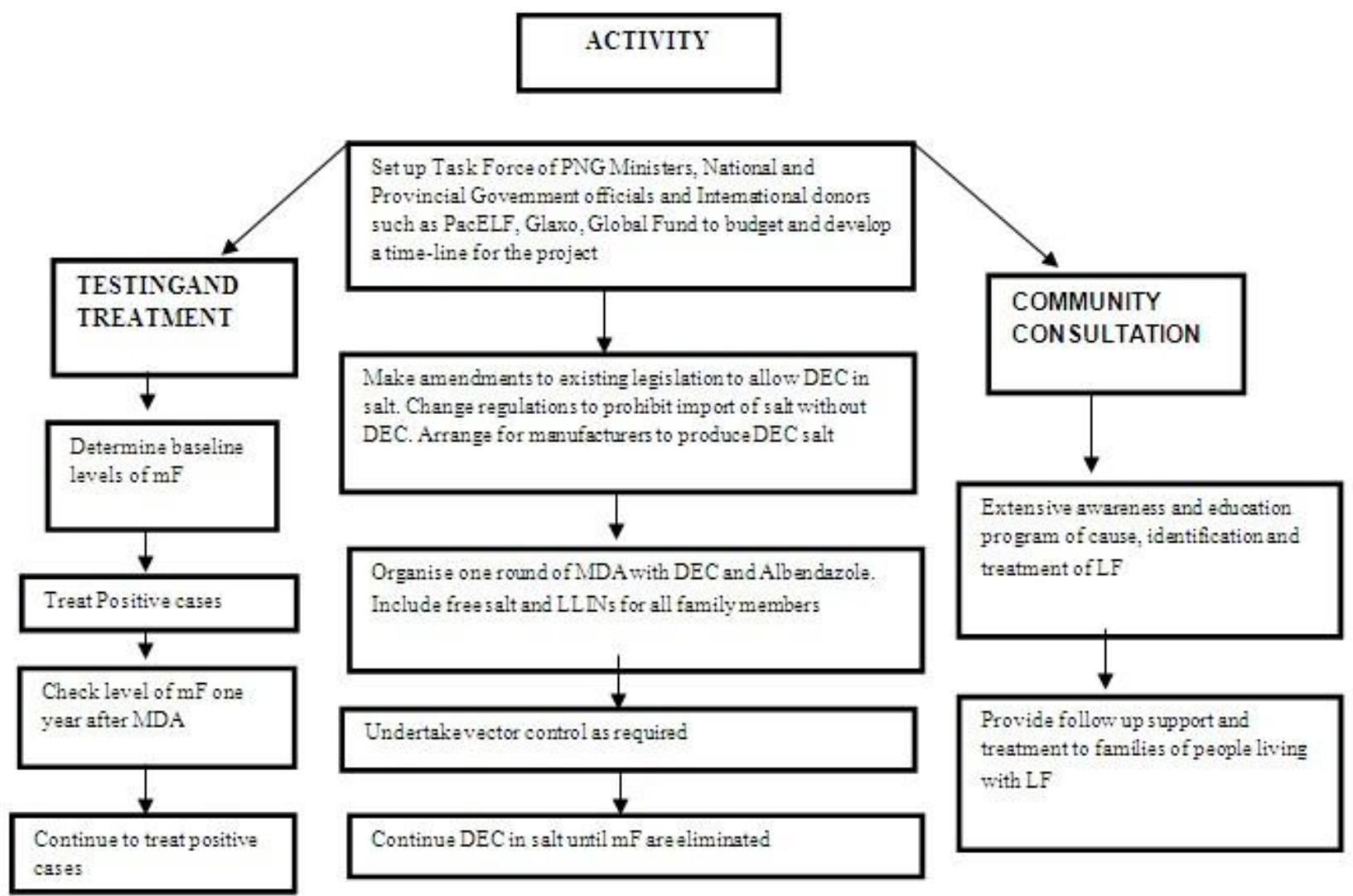

Figure 2. Suggested path for elimination of LF in PNG

\section{Conclusion}

There is no one right course of action to eliminate filariasis. A collection of strategies must be developed by each country to suit their population and topography, the vector or vectors involved co-infection with other parasites and the infrastructure available to deliver treatment. In PNG, an integrated program using MDA, DEC-fortified salt, community awareness and vector control should be adopted to replace the present government plan and urgent action taken to bring appropriate treatment to people suffering from LF. The pathway for elimination of LF in PNG is as suggested in Figure 2 below. While more research is needed in many areas, this should not delay or compromise the nationwide LF elimination program or access to treatment.

\section{Competing Interests:}

We declare that we have no competing interests. All work was done through literature review and there was no financial contribution received. 


\section{List of Abbreviations}

DEC: Diethylcarbamazine

GPELF: Global Programme to Eliminate Lymphatic Filariasis

GAELF: Global Alliance to Eliminate Lymphatic filariasis

NDOH: National Department of Health

LF: Lymphatic Filariasis

LLIN: Long Life Impregnated Nets

IRS: Indoor Residual Spraying

MDA:Mass Drug Administration

PacELF: the Pacific Programme to Eliminate

Lymphatic filariasis

PNG: Papua New Guinea

PNGELF: National Strategic Plan to Eliminate

Lymphatic Filariasis 2004-2020

WHO: World Health Organisation

\section{References}

[1] Hawking, F., Marques, R.J,"Control of Bancroftian filariasis by Cooking Salt medicated with Diethylcarbamazine", Bulletin of World Health Organisation, 37, 405-414, 1967.

[2] World Health Organisation (WHO), Lymphatic filariasis Fact sheet No. 102 January 2012.

[3] Milner, T,An Investigation into the feasibility of a National Program of DEC salt for Papua New Guinea for the Elimination of the Transmission of Lymphatic Filariasis; PNG, 2007.

[4] Manguin, S., Bangs, M.J., Pothikasikorn, J., Chareonviriyaphap, T,"Review on global co-transmission of human Plasmodium species and Wuchereria bancrofti by Anopheles mosquitoes,'Infection, Genetics and Evolution, 10, 159-177, 2010.

[5] Melrose, W.D, "Lymphatic filariasis: new insights into an old disease",International Journal for Parasitology 32, 947-960, February 2002.

[6] Centers for Disease Control and Prevention, "Life Cycle of Wuchereria bancrofti".

[7] World Health Organisation (WHO), Western Pacific Region Website Country Profile.

[8] Bockarie, MJ., Ibam, E., Alexander, NDE., Hyun, P., Dimber, ZB., Bockarie, F., Alpers, MP., Kazura, JW,"Towards eliminating lymphatic filariasis in Papua New Guinea; impact of annual single-dose mass treatment on transmission of Wuchereria bancrofti in East Sepik Province,'PNG Med. Journal, 43, 172-182, 2000.

[9] Bockarie, MJ., Tisch, DJ., Kaasterns, W., Alexander, NDE., Dimber, ZB., Bockarie, F., Ibam, E., Alpers, MP., Kazura, JW, "Mass treatment to eliminate filariasis in Papua New Guinea". New England Journal of Medicine, 347(23), 1841-1848, December 2002

[10] Bryan, J.H, "Vectors of Wuchereria bancrofti in the Sepik provinces of Papua New Guinea",Transactions of the Royal Society of Tropical Medicine and Hygiene, 80, 123-131, 1986.

[11] Melrose, W., Pisters, P., Turner, P., Kombati, Z.,Selve, BP., Hii, J., Speare, R., "Prevalence of filarial antigenaemia in Papua New Guinea: results of surveys by the School of Public Health and Tropical Medicine, James Cook University , Townsville, Australia,'PNG Medical Journal, 43 (3-4),161-165, Sep-Dec 2000.

[12] Wynd, S., Melrose, WD., Durrheim, DN., Carron, J., Gyapong, M, "Understanding the community impact of lymphatic filariasis: a review of the sociocultural literature,"Bulletin of the World Health Organisation, 85(6), 493-498, June 2007.

[13] Ottesen, E.A., Duke, B.O.L., Karam, M., Behbehani, K,"Strategies and tools for the control/elimination of lymphatic filariasis,"Bulletin of the World Health Organisation, 75 (6): 491503, 1997.

[14] Papua New Guinea, Department of Health: National Strategic Plan to Eliminate Lymphatic filariasis, PNG, 2004-2020, 2007.

[15] Haddix, A.C., Kestler, A., "Elimination of lymphatic filariasis as a public health problem: Lymphatic filariasis: economic aspects of the disease and programmes for its elimination, Transactions of the Royal Society of Tropical Medicine and Hygiene, 94, 592-593, July 2000.

[16] World Health Organisation (WHO), Global Malaria Programme WHO and DDT for Malaria Control-June 2009.

[17] Sapak, P., Melrose, W., Durrheim, D., Pawa, F., Wynd, S., Leggat, P., Taufa, T., Bockarie, M, Evaluation of the Lymphatic Filariasis Control Program, Samarai Murua District, Papua New Guinea, James Cook University, 2004.

[18] Wynd, S., Durrheim, DN., Carron, J.,Selve, B., Chaine, JP., Leggat, PA., Melrose, W, "Socio-cultural insights and lymphatic filariasis control-lessons from the Pacific".Filaria Journal, 6(3), February 2007.

[19] Gasparyan,A.Y.,Ayvazya, L., Blackmore,H., Kitas, G.D, "Writinganarrative biomedical review: considerations for authors, peer reviewers, and editors, "Rheumatol Int,31,1409-1417,2011.

[20] Gyapong, M., Gyapong, J.O., Owusu-Banalene, G., "Communitydirected treatment the way forward to eliminating lymphatic filariasis as a public health problem in Ghana",Ann Trop Med Parasitology, 95, 77-86,2001.

[21] Nandha, B., Krishnamoorthy, K., "School-based health education campaign - a potential tool for social mobilization to promote the use of DEC-fortified salt towards elimination of lymphatic filariasis,"Health Education Research, 22(4), 539-545, 2007.

[22] Fernando, S.D., Rodrigo, C., Rajapakse, S., "Current evidence on the use of antfilarial agents in the management of bancroftian filariasis,"Journal of Tropical Medicine.

[23] Leggat, P.A., Melrose, W.D., Durrheim, D.N., "Could it be Lymphatic Filariasis?" Journal of Travel Medicine, 11, 56-60, 2004.

[24] Ottesen, E. A., "The Global Programme to Eliminate Lymphatic Filariasis,"Tropical Medicine and International Health, 5(9), 591594, September 2000.

[25] Sapak, P., Williams, G, "The influence of bed nets on Bancroftian Filariasis in Buhutu Valley, Papua New Guinea", Pacific Health Dialogue, 4(1), 35-38, 1997

[26] Molyneux, D, "Lymphatic Filariasis (Elephantiasis) Elimination: A public health success and development opportunity," Filaria Journal, 2: 13, September 2003.

[27] Kazura, JW., Bockarie, MJ, "Lymphatic filariasis in Papua New Guinea: interdisciplinary research on a national health problem,"Trends in Parasitology, 19(6), 260-263, June 2003.

[28] Weil, G.J., Kastens,W., Susapu, M., Laney, S.J., Williams, S.A., King, C.L., Kazura, J.W., Bockarie, M.J, "The Impact of Repeated Rounds of Mass Drug Administration with Diethylcarbamazine plus Albendazole on Bancroftian Filariasis in Papua New Guinea," Neglected Tropical Diseases, 2(12), e344.

[29] Gelband, H, "Diethylcarbamazine salt in the control of lymphatic filariasis," American Journal of Tropical Medicine and Hygiene, 50(6), 655-662, 1994.

[30] Zhongguo, J.S., Chong, X.Y., Yu, J.C., Sheng, C., Bing, Z.Z, “A great success in lymphatic control in China. National Technical Steering Group for Filariasis Control and Research,"Chinese Journal of Parasitology and Parasitic Disease, MOPH, PMID 7554168, 1995.

[31] Adinarayanan, S., Critchley, J.A., Das, P.K., Gelband, H, "Diethylcarbamazine (DEC)-medicated salt for community-based control of lymphatic filariasis," Cochrane Database of Systematic Reviews, 2007,1.

[32] Ichimori, K., Graves, PM., Crump, A, "Lymphatic filariasis elimination in the Pacific: PacELF replicating Japanese success," Trends in Parasitology, 23(1), 36-40, November 2006.

[33] Melrose, W, Deforestation in Papua New Guinea: Potential Impact on Health Care School of Public Health and Tropical Medicine, James Cook University; 1999.

[34] Boreham, P., Marks, E., "Human filariasis in Australia: introduction, investigation and elimination,"Proceedings of the Royal Society of Queensland, 97, 23-52, 1986.

[35] Bryan, J.H., Southgate, B., "Factors affecting transmission of Wuchereria bancrofti by anopheline mosquitoes. Uptake of microfilariae," Transactions of the Royal Society of Tropical Medicine and Hygiene, 82, 128-137, 1988

[36] Encyclopedia Smithsonian: Pheromones in Insects http://www.si.edu/Encyclopedia_SI/nmnh/buginfo/pheromones.ht $\mathrm{m}$.

[37] Bray, DP, Bandi, KK, Brazil, RP, Oliveira, AG, Hamilton, JGC, "Synthetic Sex Pheromone Attracts the Leishmaniasis Vector Lutzomia longipalpis (Diptera: Psychodidae) to Traps in the Field”, J Med Entomol, 46(3), 428-434, May 2009. 
[38] Burkot, TR., Ichimori, K,'The PacELF programme: will mass drug administration be enough?" Trends inParasitology, 18(3),109-115, March 2002.

[39] Charlwood, D., Dagoro, H, "Impregnated bednets for the control of filariasis transmitted by Anopheles punctulatus in rural Papua New Guinea”, PNG Medical Journal, 30, 199-202, 1987.

[40] World Health Organisation (WHO), Global Malaria Programme WHO and DDT forMalaria Control-June 2009.

[41] Alexander, N.D.E., Bockarie, M.J., Dimber, Z.B., Griffin, L., Kazura, J.W., Alpers, M.P, "Migration and dispersal of lymphatic filariasis in Papua New Guinea," Transactions of the Royal Society of Tropical Medicine and Hygiene, 95, 277-279, 2001.

[42] Burkot, TR., Durrheim, DN., Melrose, WD., Speare, R., V., Ichimori, K,“ The argument for integrating vector control with multiple drug administration campaigns to ensue elimination of lymphatic filariasis",Filaria Journal, 5(10), August 2006.

[43] Ramaiah, K.D., Das, P.K., Vanamail, P., Pani, S.P, "Impact of 10 years of diethylcarbamazine and ivermectin mass administration on infection and transmission of lymphatic filariasis,"Transactions of the Royal Society of Tropical Medicine and Hygiene, 101(8), 555-563, March 2007.
[44] Huppatz, C., Durrheim, D., Lammaie, P., Kelly, P., Melrose, W., "Eliminating lymphatic filariasis- the surveillance challenge," Tropical Medicine and International Health, 13(3), 292-294, March 2008.

[45] Esterre, P., Plichart, C., Sechan, Y., Nguyen, NL, "The impact of 34 years of massive DEC chemotherapy on Wuchereria bancrofti infection and transmission: the Maupiti cohort," Journal of Tropical Medicine and International Health, 16(3), 190-195, March 2001

[46] Noroes, J., Dreyer, G., Santos, A., Mendes, VG., Medeiros, Z., Addiss, D.,"Assessment of the efficacy of diethylcarbamazine on adult Wuchereria bancrofti in vivo," Transactions of the Royal Society of Tropical Medicine and Hygiene, 91, 78-81,1997.

[47] Lahariya, C., Tomar, S.S, "How endemic countries can accelerate lymphatic filariasis elimination? An analytical review to identify strategic and programmatic interventions," Journal Vector Borne Diseases, 48, 1-6,March 2011

[48] Tisch, DJ., Michael, E., Kazura, J.W, "Mass chemotherapy options to control lymphatic filariasis: a systematic review," The Lancet, 5, 514-523,August 2005.

[49] Fan, P.C,"Filariasis eradication on Kinmen Proper, Kinmen (Quemoy) Islands, Republic of China,"Acta Tropica, 47(3),161169,1990 . 